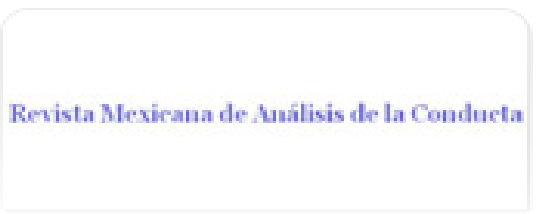

Revista Mexicana de Análisis de la Conducta ISSN: 0185-4534

editora@rmac-mx.org

Sociedad Mexicana de Análisis de la Conducta

México

HERNÁNDEZ, VARSOVIA; BRUNER, CARLOS A.

EFECTOS DEL INTERVALO ESTÍMULO-COMIDA EN COMBINACIÓN CON LA DURACIÓN DEL PERIODO SIN ACCESO A LA COMIDA SOBRE LA INGESTA DE ALIMENTO EN RATAS

Revista Mexicana de Análisis de la Conducta, vol. 39, núm. 3, diciembre-, 2013, pp. 1-14

Sociedad Mexicana de Análisis de la Conducta

Distrito Federal, México

Disponible en: http://www.redalyc.org/articulo.oa?id=59335809001

Cómo citar el artículo

- Número completo

- Más información del artículo

- Página de la revista en redalyc.org

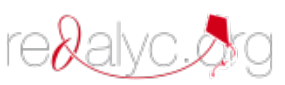

Sistema de Información Científica

Red de Revistas Científicas de América Latina, el Caribe, España y Portugal Proyecto académico sin fines de lucro, desarrollado bajo la iniciativa de acceso abierto 


\title{
EFECTOS DEL INTERVALO ESTÍMULO-COMIDA EN COMBINACIÓN CON LA DURACIÓN DEL PERIODO SIN ACCESO A LA COMIDA SOBRE LA INGESTA DE ALIMENTO EN RATAS
}

\author{
EFFECTS OF THE STIMULUS-FOOD INTERVAL IN COMBINATION \\ WITH THE DURATION OF THE PERIOD WITHOUT ACCESS TO \\ FOOD ON FOOD INTAKE BY RATS
}

\author{
VARSOVIA HERNÁNDEZ Y CARLOS A. BRUNER \\ FACULTAD DE PSICOLOGÍA \\ UNIVERSIDAD NACIONAL AUTÓNOMA DE MÉXICO
}

\section{Resumen}

Relativo a una oportunidad de comer no señalada, una señal contigua que precede al subsecuente acceso a la comida produce un incremento en la cantidad de comida consumida por ratas. En este experimento tres ratas permanecieron en las cámaras experimentales durante 24 horas al día, siete días a la semana. El intervalo entre una señal de 5 minutos y la subsecuente oportunidad de comer de 10 minutos se varió sistemáticamente en 5, 10 ó 20 minutos. Esta manipulación se combinó con periodos sin acceso a la comida de 20 ó 300 minutos. En comparación con una línea base no señalada, la presentación del estímulo a los 5 minutos (contigua) incrementó la ingesta durante el periodo sin acceso a la comida de 20 minutos y aún más durante el periodo de 300 minutos. Alargar el intervalo estímulo-comida disminuyó la ingesta gradualmente bajo ambos periodos sin accesoa la comida, incluso por debajo de línea base. Dichos resultados son discu-

\footnotetext{
Varsovia Hernández y Carlos A. Bruner, Laboratorio de Condicionamiento O perante, Facultad de Psicología, U niversidad Nacional Autónoma de México.

Los datos de este artículo forman parte de la tesis de doctorado del primer autor cuyos estudios fueron apoyados por la beca 223216 del CONACYT.

Dirigir correspondencia a Varsovita Hernández, Laboratorio de Condicionamiento O perante, 2ㅇiso, Facultad de Psicología, U niversidad Nacional Autónoma de México. Av. U niversidad 3004, Col. CopilcoUniversidad, México, D.F. C.P. 04510. Correo electrónico: varsovia07@gmail.com.
} 
tidos en términos de la función intensificadora o supresora de la ingesta de un estímulo neutral.

Palabras clave: estímulo neutral, periodo de acceso a la comida, periodo sin acceso a la comida, ingesta de comida, ratas.

\begin{abstract}
Relative to an unsignaled feeding opportunity, a contiguous signal that precedes the subsequent access to food increases the amount of food consumed by rats. In this experiment three rats remained in the experimental chamber for 24 hours a day, seven days a week. The interval between a 5-min signal and a subsequent 10-min opportunity to eat was in different conditions 5, 10 or $20 \mathrm{~min}$. This variable was combined with periods without access to food of either 20 or $300 \mathrm{~min}$. In comparison to an unsignaled baseline, the 5-min (contiguous) signal increased eating during the 20-min period without access to food and even more during the 300 -min period. Lengthening the stimulus to food interval gradually decreased eating under both no-food-access periods even below baseline. These results were discussed in terms of the eating-enhancing and eating-suppressing functions of a neutral stimulus.

Keywords: neutral stimulus, food-access period, period without access to food, food intake, rats.

Un hallazgo que ha sido reportado en diferentes estudios es que manteniendo constante el nivel de privación de alimento, presentar un estímulo en contigüidad con un periodo de acceso a la comida controla una mayor ingesta en ratas en comparación con una situación en la que no se presenta el estímulo (Hernández \& Bruner, 2012; Lovibond, 1980; Zamble, 1973). Los autores de estos trabajos explicaron sus resultados de diferentes formas, por ejemplo, indicando que estímulos que se presentan en contigüidad con la comida adquieren la función de incentivos condicionados que energizan la ingesta (Lovibond, 1980; Zamble, 1973).

Con el fin de investigar si la ingesta de comida controlada por la presentación contigua del estímulo es sensible a la manipulación del intervalo estímulo-comida, Hernández y Bruner (2012) realizaron un estudio en el que variaron dicho intervalo. Esta manipulación se realizó debido a que tanto en estudios de condicionamiento respondiente como de condicionamiento operante se encontraron cambios en la magnitud o tasa de respuesta en función de variar el intervalo entre la presentación de un estímulo y el periodo de acceso a la comida o al reforzador (e.g., Farmer \& Schoenfeld, 1966; Pavlov, 1927). Los sujetos fueron tres ratas que vivieron en las cámaras experimentales durante todo el estudio. Durante la fase de línea base se presentaron periodos de acceso a la comida de 10 minutos separados por periodos sin acceso de 160 minutos. Durante el periodo de acceso cada presión a una palanca
\end{abstract}


produjo una bolita de comida. En fases sucesivas se introdujo un tono de 5 minutos a intervalos de 5, 10, 20, 40, 80 y 160 minutos con respecto al siguiente periodo de acceso a la comida. Encontraron que presentar el estímulo 5 minutos antes del periodo de acceso a la comida controló aumentos en la cantidad de comida consumida mientras que alargar el intervalo estímulo-comida más allá de 10 minutos controló disminuciones progresivas en la ingesta, incluso por debajo del nivel de línea base.

La importancia del estudio de Hernández y Bruner (2012) radica en que mostró que manteniendo constante el periodo sin acceso a la comida es posible controlar tanto aumentos como disminuciones en la ingesta al variar el intervalo entre la presentación de un estímulo y el periodo de acceso a la comida, lo cual no se había reportado con anterioridad.

En el estudio de Hernández y Bruner fue necesario mantener fijo el periodo sin acceso a la comida debido a que la ingesta varía en función de la duración de dicho periodo (Díaz, 2008), lo cual hubiera confundido el efecto de la presentación de estímulos con el de la privación de comida. Debido a que la duración del periodo sin acceso a la comida y la presentación del estímulo en diferentes ubicaciones del periodo sin acceso son dos operaciones que afectan la ingesta, es importante estudiar de qué manera interactúan para controlar la cantidad de comida ingerida.

Referente al efecto de variar la duración de los periodos sin acceso a la comida sobre la ingesta, el consenso en la literatura es que la cantidad de comida ingerida durante un periodo de acceso varía en función de la duración del periodo sin acceso anterior a la comida, a la manera de un periodo de privación (Baker, 1955; Díaz, 2008; Siegal, 1961). Por ejemplo, Díaz (2008) realizó un estudio en el que en sesiones de 24 horas mantuvo fijo el periodo de acceso a la comida en 10 minutos y varió la duración del periodo sin acceso en 45, 180 y 720 minutos. Los sujetos obtuvieron una bolita de comida durante el periodo de acceso al presionar una palanca. Díaz encontró que la ingesta de comida aumentó al alargar la duración del periodo sin acceso.

Con respecto a la interacción entre la duración del periodo sin acceso y la presentación de estímulos sobre la ingesta, esta relación se abordó sólo en un estudio (Zamble, 1973, Experimento 2). Zamble determinó el efecto de presentar un estímulo antecedente al acceso a la comida sobre la ingesta. A un grupo de ratas le presentó un estímulo 15 minutos antes de un periodo de acceso a la comida de 30 minutos y a un segundo grupo le presentó el estímulo después de la presentación del periodo de acceso a la comida. Para ambos grupos el periodo sin acceso a la comida varió entre 4 y 44 horas teniendo como promedio 24 horas. Zamble encontró que el grupo de sujetos con la presentación del estímulo antecedente al periodo de acceso ingirió más comida que el grupo con la presentación del estímulo posterior al acceso. Al analizar los datos de la ingesta en función de la duración del periodo sin acceso anterior, se encontró para ambos grupos que a mayor duración del periodo sin acceso fue mayor la cantidad de comida ingerida, teniendo como 
máximo 28 horas. A partir de ese periodo se encontraron disminuciones en la ingesta. Adicionalmente, Zamble encontró que la diferencia en la cantidad de comida ingerida entre ambos grupos fue mayor conforme alargó la duración del periodo sin acceso a la comida. No obstante, no reportó la magnitud de dichas diferencias.

En el estudio de Zamble se reportó la ingesta en función de la duración del periodo sin acceso a la comida y de la presentación contigua de un estímulo antecedente a la comida, sin embargo se desconoce el efecto de variar la ubicación del estímulo en combinación con variar la duración del periodo sin acceso a la comida. Una manera de investigar esto es manipulando la duración del periodo sin acceso a la comida en relación a la duración de 160 minutos utilizado en el estudio de Hernández y Bruner (2012). Teniendo como base la duración del periodo sin acceso de 160 minutos se puede determinar el efecto de alargar o acortar la duración de este periodo en combinación con el efecto de alargar el intervalo entre la presentación del estímulo y el acceso a la comida. Por lo tanto, el propósito de este experimento fue determinar el efecto de variar la duración del periodo sin acceso a la comida con respecto a los 160 minutos utilizados en el estudio de Hernández y Bruner (2012) en combinación con el efecto de variar el intervalo entre la presentación de un estímulo y el periodo de acceso a la comida sobre la cantidad de comida consumida. Adicionalmente, la realización de este estudio permitió determinar si es posible replicar el hallazgo de Hernández y Bruner referente a disminuciones en la ingesta de comida al alargar el intervalo estímulo-comida.

\section{Método}

\section{Sujetos}

Se utilizaron tres ratas W istar macho de cuatro meses de edad y experimentalmente ingenuas. Durante todo el estudio los sujetos vivieron en las cámaras experimentales. El acceso al agua se mantuvo libre durante todo el estudio mientras que el tiempo de acceso a la comida fue de 80 minutos por día.

\section{Aparatos}

Se utilizaron tres cámaras experimentales de $49 \mathrm{~cm}$ de ancho $\times 40 \mathrm{~cm}$ de altura $\mathrm{x}$ $38 \mathrm{~cm}$ de profundidad. En el interior de cada cámara se instaló un panel frontal en el que se colocó una palanca (M odelo EN V-110RM, M ed Associates Inc.) sensible a una fuerza de $0.15 \mathrm{~N}$. La palanca se ubicó a un altura de $6.5 \mathrm{~cm}$ respecto del piso de la caja y a $2 \mathrm{~cm}$ respecto de la pared izquierda de la caja. A la derecha de la palanca se colocó un comedero de metal el cual se ubicó $3.5 \mathrm{~cm}$ por detrás del nivel del panel y a $3.5 \mathrm{~cm}$ respecto del piso de la caja. En la parte posterior del comedero se conectó un dispensador de comida (M odelo EN V-203IR, M ed Associates Inc.) que sirvió para 
entregar bolitas de comida de $25 \mathrm{mg}$. Las bolitas de comida se fabricaron remoldeando comida para ratas de la marca Zeigler. En la parte posterior del panel frontal se colocó una pipeta que se encontraba recedida $0.7 \mathrm{~mm}$ dentro de la cámara experimental y permitía el libre acceso al agua. La pipeta estaba conectada a una botella con agua que se encontraba al otro lado del panel. La pipeta se ubicó a $19.5 \mathrm{~cm}$ a la derecha de la palanca y a $8 \mathrm{~cm}$ respecto del piso de la caja. En el panel frontal también se colocó un generador de sonido (ENV-223AM) que produjo un tono de $60 \mathrm{~dB}$ y se ubicó $22 \mathrm{~cm}$ por arriba del comedero. Para simular el ciclo de luz permanecieron encendidos dos focos de $28 \mathrm{v}$ los cuales se ubicaron a $28 \mathrm{~cm}$ respecto del piso de la caja y estaban separados $18 \mathrm{~cm}$ entre sí. Cada caja estaba equipada con un ventilador que facilitó la circulación de aire y también sirvió para enmascarar ruidos ajenos a la investigación. El registro y control de los eventos experimentales se realizó por medio de una computadora con software M ED-PC IV acoplada a una interfase M ed-Associates Inc. (Modelo SG -503), la cual se ubicó en un cuarto adyacente al lugar en el que se encontraban las cámaras experimentales.

\section{Procedimiento}

Todas las sesiones tuvieron una duración de 22 horas con 40 minutos. El ciclo de luz estuvo vigente de las 9:00 am a 8:20 pm y el ciclo de oscuridad se mantuvo de las 8:20 pm a 7:40 am. El complemento de las 22 horas con 40 minutos se ocupó en pesar y rellenar las botellas con agua, verificar el funcionamiento de los dispensadores de comida, rellenarlos y limpiar las cámaras experimentales. El acceso al agua fue de manera libre durante todo el estudio. En ninguna de las fases del experimento se encontraron residuos de comida en los comederos o en el piso de las cámaras experimentales.

Línea base. Con el fin de establecer la respuesta de presión a la palanca, durante 5 días se expuso directamente a los sujetos a un programa de reforzamiento de razón fija conforme al cual por cada presión a la palanca se entregó una bolita de comida (razón fija 1, RF1).

Como se muestra en la Figura 1, cada día se programaron ocho periodos de acceso a la comida con duración de 10 minutos. Durante los periodos de acceso los sujetos obtuvieron una bolita de comida por cada presión a la palanca. Los periodos de acceso a la comida fueron señalados con presentaciones de un tono intermitente. El tono intermitente consistió del encendido y apagado del generador de tonos cada segundo. Los periodos de acceso se separaron por periodos sin acceso que podían tener una duración de 20 minutos (corta) o una duración de 300 minutos (larga). Las duraciones de los periodos sin acceso alternaron al azar con la restricción de que cada uno ocurriera cuatro veces por día. En promedio la duración de ambos periodos sin acceso fue de 160 minutos, la cual fue la duración utilizada en el estudio de Hernández y Bruner (2012). Durante toda la sesión experimental 
se registraron las presiones a la palanca. La línea base se mantuvo vigente durante 30 sesiones.
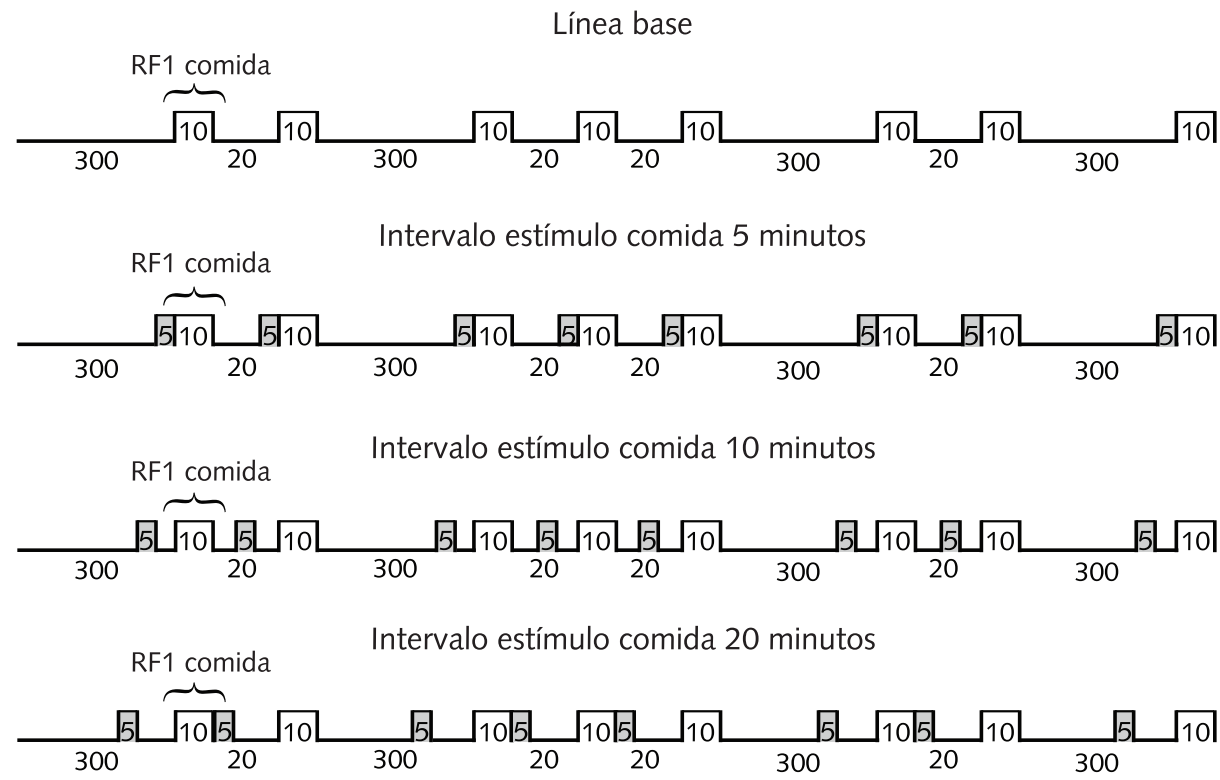

Figura 1. Esquema del procedimiento utilizado. Las duraciones presentadas se encuentran en minutos. RF1 comida indica que se encuentra en vigencia un programa de razón fija 1 conforme al cual durante 10 minutos se entregó una bolita de comida por cada presión a la palanca, en estos 10 minutos se presentó un tono intermitente. Los periodos sin acceso a la comida fueron de 20 ó 300 minutos y ocurrieron al azar. En la fase de línea base no se presentó ningún estímulo dentro del periodo sin acceso a la comida. Para las fases posteriores se muestran las diferentes posiciones del estímulo intrusivo dentro del periodo sin acceso a la comida, el estímulo consistió de un tono constante que tuvo una duración de 5 minutos.

Introducción del estímulo. Manteniendo constantes las condiciones de la línea base, en fases de 30 días se introdujo un tono continuo en una ubicación temporal diferente dentro de cada periodo sin acceso. En cada fase la ubicación del tono continuo se mantuvo fija y tuvo una duración de 5 minutos. En la primera fase se presentó el tono continuo cinco minutos antes del periodo de acceso, por lo que al final de los cinco minutos de presentación del tono dio inicio el periodo de acceso a la comida. En fases sucesivas, el intervalo entre el inicio de la presentación del tono 
continuo y el inicio del periodo de acceso a la comida se alargó a 10 y 20 minutos. El intervalo más largo fue de 20 minutos ya que fue el máximo intervalo permitido por la duración del periodo sin acceso corto. Después de introducir al tono en la ubicación de 20 minutos se redeterminó el efecto de presentarlo en la posición de 5 minutos.

\section{Resultados}

Para los tres sujetos la respuesta de presión a la palanca que era seguida de la entrega de una bolita de comida se estableció en la primera sesión.

Para todas las figuras los resultados se muestran como el promedio de las últimas 10 sesiones para la línea base y para los diferentes intervalos est́mulo-comida. Con fines de comparación en cada figura se muestran por separados los datos en función del periodo de luz y oscuridad ya que esta es una variable que afecta la ingesta de alimento en ratas (Díaz, 2008). Se decidió tomar el promedio de las últimas 10 sesiones con el fin de hacer comparables los resultados del presente estudio con los de Hernández y Bruner (2012) ya que estos autores analizaron sus datos empleando ese número de sesiones.

En la Figura 2 se muestra el promedio y desviación estándar del número de bolitas de comida obtenidas por cada sujeto durante los 80 minutos diarios de acceso a la comida en función de los diferentes periodos sin acceso antecedentes y de los diferentes intervalos estímulo-acceso. En cada panel de la figura se presenta como una línea punteada el nivel de la fase de línea base en la que no se presentó el estímulo durante los periodos sin acceso.

Durante el periodo de luz después del periodo sin acceso de 20 minutos se encontró para los tres sujetos que la cantidad de bolitas de comida obtenidas con el intervalo entre la presentación del estímulo y el acceso a la comida de 5 minutos fue similar a la de línea base. Alargar el intervalo estímulo- comida a 10 y 20 minutos no tuvo efecto en el número de bolitas de comida obtenidas para los sujetos S11 y S12, mientras que para S10 el promedio disminuyó. Para el periodo sin acceso de 300 minutos se encontró en los tres sujetos que el número de bolitas de comida obtenidas con el intervalo entre la presentación del estímulo y el acceso a la comida de 5 minutos fue mayor que en la línea base. Alargar el intervalo estímulo-comida a 10 y 20 minutos resultó en disminuciones en el número de bolitas de comida obtenidas para los tres sujetos. Al comparar el número de bolitas de comida obtenidas posterior a los periodos sin comida de 20 y 300 minutos durante la luz se encontró un ligero aumento después del periodo sin acceso largo.

Durante el periodo de oscuridad, después del periodo sin acceso de 20 minutos se encontró para los tres sujetos que la cantidad de bolitas de comida obtenidas con el intervalo entre la presentación del estímulo y el acceso a la comida de 5 minutos fue mayo que en la línea base. Alargar el intervalo estímulo-comida a 10 y 20 minutos resultó en disminuciones en la cantidad de bolitas de comida obtenidas con respecto al nivel de línea base. También durante el periodo de oscuridad, para el periodo sin acceso 


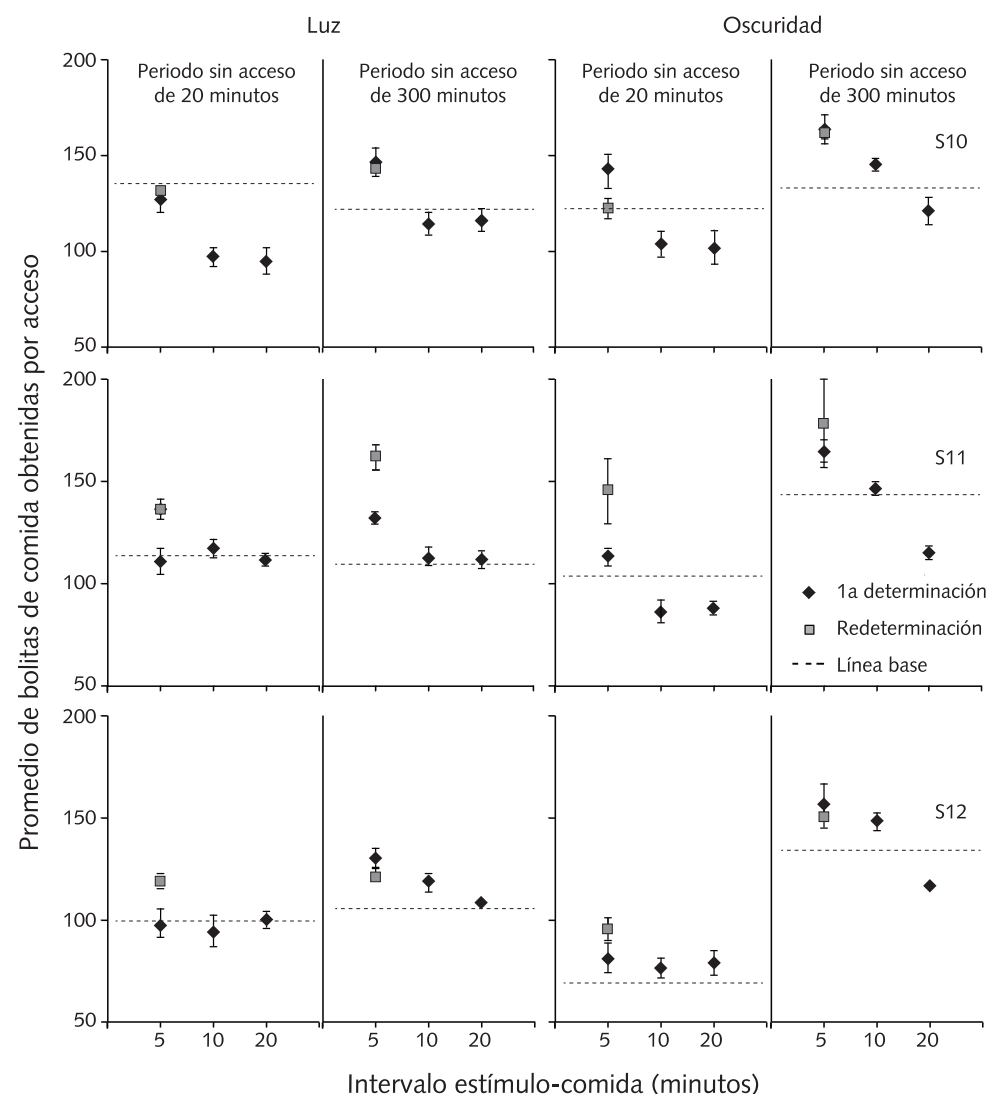

Figura 2. Promedio de bolitas de comida obtenidas por acceso por los tres sujetos para la línea base y los diferentes intervalos estímulo-acceso a la comida. Se muestran los datos en función de la duración del periodo sin acceso de 20 y 300 minutos separados para luz y oscuridad.

de 300 minutos se encontró en los tres sujetos que la cantidad de bolitas de comida obtenidas con el intervalo entre la presentación del estímulo y el acceso a la comida de 5 minutos fue mayor que en la línea base. El efecto de alargar el intervalo estímulocomida a 10 y 20 minutos fue la disminución en el número de bolitas de comida obtenidas con respecto al nivel de línea base. Al comparar las comidas obtenidas posterior a los periodos sin acceso a la comida de 20 y 300 minutos duranteoscuridad se encontró un mayor número de bolitas de comida obtenidas después del periodo sin acceso largo.

Para todos los sujetos se encontró que redeterminar el intervalo estímulo-comida de 5 minutos controló que el número de bolitas de comidas obtenidas fuera similar 
al obtenido la primera vez que se expuso a los sujetos a esta condición. Al comparar las bolitas de comida obtenidas durante periodos de luz y oscuridad se encontró para los tres sujetos que el nivel de bolitas de comida obtenidas fue más elevado durante el periodo de oscuridad en comparación con el de luz.

Con el mismo formato que la Figura 2, en la Figura 3 se muestra la latencia entre el inicio del periodo de acceso a la comida y la primer respuesta por comida en función de los diferentes periodos sin acceso y de los diferentes intervalos estímulo-comida. La latencia se muestra como el promedio de las ocho oportunidades de comer que ocurrieron en el periodo de 24 horas junto con sus respectivas desviaciones estándar.

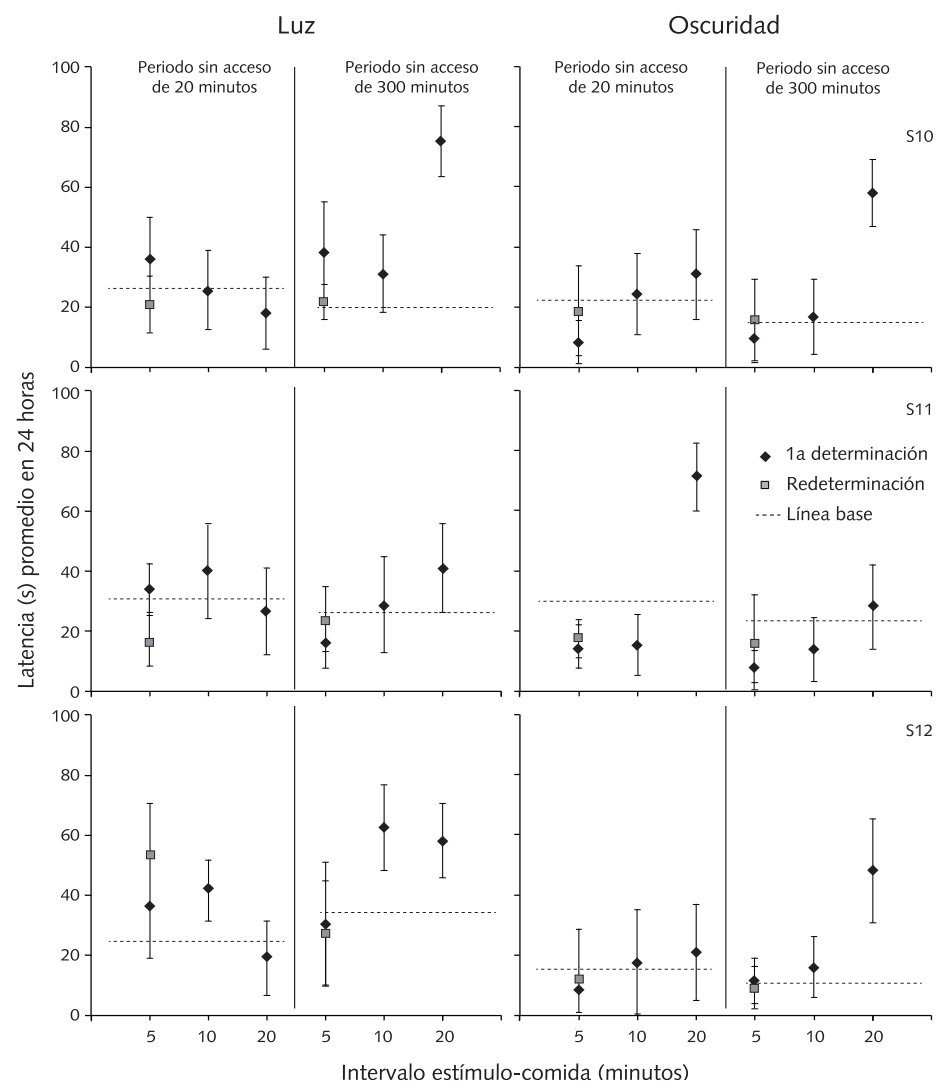

Figura 3. Latencia promedio entre el inicio del periodo de acceso a la comida y la primer respuesta por comida para los diferentes intervalos estímulo-acceso a la comida. Se muestran los datos en función de la duración del periodo sin acceso de 20 y 300 minutos separados para luz y oscuridad. 
En el periodo de luz y después del periodo sin acceso de 20 minutos no se encontró un efecto claro de presentar un estímulo durante el periodo sin acceso y alargar el intervalo estímulo-comida sobre la duración de la latencia para los tres sujetos. En el periodo sin acceso de 300 minutos durante el periodo de luz para el sujeto S10 el efecto de introducir el estímulo en la ubicación de 5 minutos con respecto al siguiente periodo de acceso a la comida fue un aumento en la duración de latencia, mientras que para los sujetos S11 y S12 introducir el estímulo en esa ubicación controló una disminución de la duración de la latencia con respecto al nivel de línea base. Para estos dos sujetos, alargar el intervalo estímulo-comida tuvo el efecto de aumentar la duración de la latencia con respecto al nivel de línea base mientras que para S10 no hubo un efecto claro.

Durante el periodo de oscuridad, después del periodo sin acceso a la comida de 20 minutos para los tres sujetos se encontró que la duración de la latencia fue menor con el intervalo estímulo-comida de 5 minutos en comparación con el nivel de línea base. Alargar el intervalo estímulo-comida controló ligeros aumentos en la duración de la latencia con respecto al nivel de línea base. En el periodo sin acceso de 300 minutos durante oscuridad se encontró para los tres sujetos que la duración de la latencia fue menor durante la presentación del estímulo con el intervalo de 5 minutos al periodo de acceso a la comida en comparación con la línea base. Alargar el intervalo estímulo-comida controló aumentos progresivos en la duración de la latencia. Al realizar una inspección visual de los datos no se encontraron diferencias claras en la duración de la latencia entre el periodo sin acceso a la comida de 20 y 300 minutos ni tampoco entre los periodos de luz y oscuridad.

Con respecto al peso de los sujetos, éste no varió no varió de forma sistemática durante los diferentes intervalos estímulo comida siendo la media de su peso en gramos de $416(D E=9.9), 352.4(D E=12.5)$ y $399(D E=6.1)$ para los sujetos S10, S11 y $\mathrm{S} 12$, respectivamente.

Debido a que en este estudio se tomó como base la duración del periodo sin acceso a la comida de 160 minutos utilizada en el estudio de Hernández y Bruner (2012) para determinar el efecto de aumentar o disminuir la duración de ese periodo, en la Figura 4 se muestra el promedio y desviación estándar del número de bolitas de comida obtenidas por acceso para los tres sujetos en función de los periodos sin acceso antecedentes de 20 y 300 minutos utilizados en el presente experimento y también para el periodo sin acceso de 160 minutos del estudio de Hernández y Bruner. En ese estudio el método empleado fue el mismo que en el presente trabajo con la diferencia de que la duración del periodo sin acceso se mantuvo fija y se determinó el efecto de un mayor número de ubicaciones del estímulo con respecto al siguiente periodo de acceso a la comida. Al igual que en la figura anterior, el número de bolitas de comida obtenidas durante la línea base se muestra con una línea punteada.

Durante el periodo de luz se encontró que el efecto de aumentar el periodo sin acceso en combinación con la presentación del estímulo en el intervalo estímulo- 


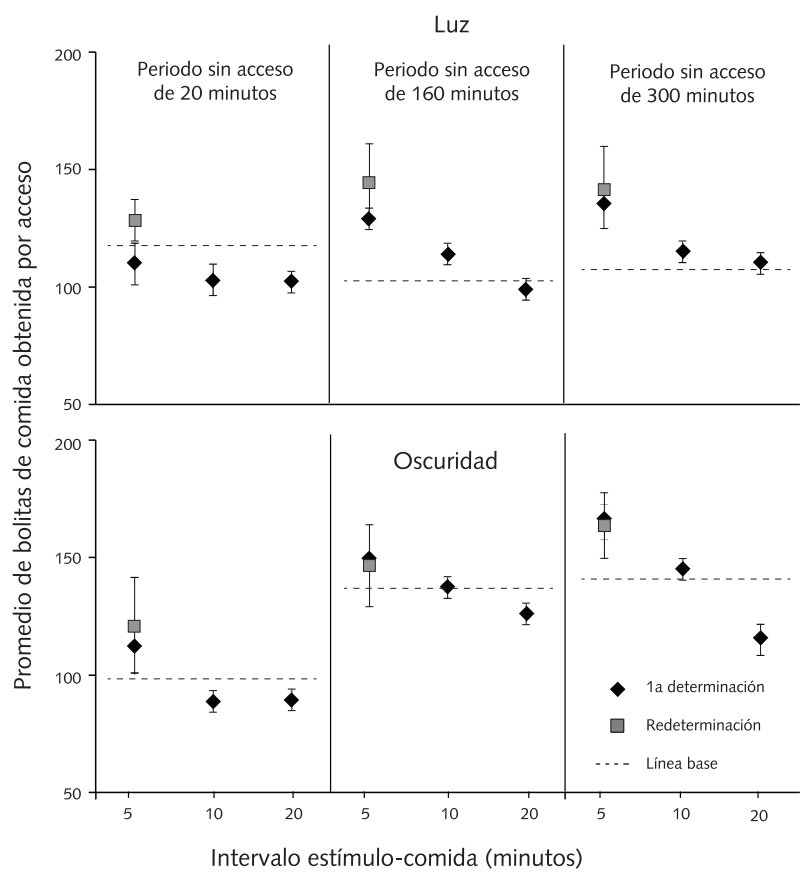

Figura 4. Bolitas de comida obtenidas por acceso como promedio de los tres sujetos para la línea base y los diferentes intervalos estímulo-acceso a la comida separados para luz y oscuridad. Se muestran los datos en función de la duración del periodo sin acceso de 20 y 300 minutos utilizados en este estudio y para el periodo sin acceso de 160 minutos del estudio de Hernández y Bruner (2012).

comida de 5 minutos fue un leve aumento en el promedio del número de bolitas de comida obtenidas. También durante la luz se encontró que al aumentar el intervalo estímulo-comida el promedio de bolitas de comidas obtenidas disminuyó de manera progresiva.

Para el periodo de oscuridad se encontró que aumentar el periodo sin acceso a la comida de 20 a 300 minutos controló aumentos aun mayores en el promedio de bolitas de comida obtenidas. Presentar el estímulo 5 minutos antes del periodo de acceso a la comida controló aumentos en el número de bolitas de comida obtenidas mientras que alargar el intervalo estímulo-comida controló disminuciones progresivas en el número de bolitas de comida obtenidas. Al comparar la ingesta durante los periodos de luz y oscuridad se encontró que durante el periodo de oscuridad el nivel global de las bolitas 
de comida obtenidas fue mayor. En todos los sujetos se encontró que redeterminar el intervalo estímulo-comida de 5 minutos controló un número de bolitas de comidas obtenidas similar al obtenido la primera vez que se expuso a los sujetos a esta condición.

En el estudio de Zamble (1973) se reportó que la diferencia en la cantidad de comida ingerida entre un grupo al que se le presentó el estímulo de manera aleatoria dentro del periodo sin acceso a la comida y un grupo al que se le presentó en contigüidad con el siguiente periodo de acceso a la comida aumentó en función de aumentar la duración del periodo sin acceso. En el presente estudio el porcentaje de cambio en el número de bolitas de comida obtenidas con respecto a la línea base para el intervalo estímulocomida de 5 minutos fue de 7, 14 y 21\%, para los periodo sin acceso de 20, 160 y 300 minutos respectivamente. Para el intervalo estímulo-comida de 10 minutos fue de $-9,2$ y $5 \%$, mientras que para el intervalo estímulo-comida de 300 minutos fue de $-9,-8$ y $-9 \%$ para los mismos periodos sin acceso.

\section{Discusión}

El propósito de este experimento fue determinar el efecto de variar la duración del periodo sin acceso a la comida con respecto a los 160 minutos utilizados en el estudio de Hernández y Bruner (2012) en combinación con el efecto de variar el intervalo entre la presentación de un estímulo y el periodo de acceso a la comida sobre la cantidad de comida consumida.

Se encontró que la presentación del estímulo 5 minutos antes del siguiente periodo de acceso a la comida controló aumentos en el número de bolitas de comida obtenidas sobre el nivel de línea base tanto para el periodo de 20 minutos sin acceso como para el de 300 minutos, aunque para el periodo de 20 minutos el incremento sólo se registró durante el periodo de oscuridad. El efecto de alargar el intervalo entre el estímulo y el siguiente periodo de acceso fue que el número de bolitas de comida obtenidas disminuyó de manera progresiva después de ambos periodos sin acceso. Los resultados referentes a aumentos en la ingesta en función de presentar el estímulo en contigüidad con la siguiente comida fueron reportados en estudios anteriores (e.g., Hernández \& Bruner, 2012; Lovibond, 1980; Zamble, 1973) y muestran la importancia de dicha manipulación como factor determinante de la ingesta de comida. En cuanto al resultado de disminuciones en la ingesta de comida al alargar el intervalo estímulo-comida, se pudo replicar el hallazgo descrito por primera vez en el estudio de Hernández y Bruner (2012), lo cual demuestra que es un efecto confiable.

Referente al efecto de variar la duración del periodo sin acceso a la comida se encontró que incrementar la duración del periodo sin acceso con respecto a la de 160 minutos empleada en el estudio de Hernández y Bruner (2012) controló un mayor número de bolitas de comida obtenidas en todos los intervalos. Disminuir la duración del periodo sin acceso a 20 minutos controló disminuciones en el número de bolitas de comida obtenidas también para todos los intervalos en comparación con los 
periodos sin acceso de 160 y 300 minutos. Este hallazgo es similar al de estudios previos en los que se encontró que aumentar el nivel de privación de comida controla aumentos en la ingesta (e.g., Díaz, 2008; Siegal, 1961).

0 tra variable dependiente reportada fue la latencia entre el inicio del periodo de acceso a la comida y la primera respuesta de presión a la palanca por comida. Se encontró que con la excepción del periodo sin acceso de 20 minutos durante el periodo de luz, en general presentar un estímulo contiguo con el siguiente periodo de acceso a la comida disminuyó la duración de la latencia en comparación con la línea base. Alargar el intervalo estímulo-comida controló aumentos en la duración de la latencia con respecto al nivel de línea base. Estos resultados son similares a los reportados por Hernández y Bruner (2012) y muestran que existe una relación entre la disminución en la duración de la latencia y aumentos en la ingesta de comida. De forma análoga, aumentos en la duración de la latencia se relacionaron con disminuciones en la ingesta de comida.

En cuanto al efecto de aumentar la duración del periodo sin acceso a la comida en combinación con alargar el intervalo estímulo-comida sobre el número de bolitas de comida obtenidas, se encontró que el porcentaje de la diferencia en el número de bolitas de comida obtenidas con respecto a la fase de línea base durante el intervalo estímulo-comida de 5 minutos aumentó al alargar el periodo sin acceso a la comida. Al alargar el intervalo estímulo-comida a 10 y 20 minutos la diferencia en el número de bolitas de comida obtenidas con respecto a la línea base se mantuvo en un nivel similar entre las diferentes duraciones del periodo sin acceso a la comida. El estudio del efecto de variaciones a la duración de periodos sin acceso en combinación con presentaciones de estímulos apareados con comida sólo se describió en un estudio anterior. En ese estudio Zamble (1973) encontró que aumentar el nivel de privación de comida controló que la diferencia en la ingesta aumentara en un grupo en el que el estímulo se le presentó inmediatamente antes de un periodo de acceso con respecto a un grupo al que el estímulo se le presentó de manera aleatoria en el intervalo entre comidas. En el presente estudio se encontró el mismo resultado variando la privación en un mismo día y también se añadió el hallazgo de que alargar el intervalo estímulo-comida más allá de 5 minutos en combinación con diferentes periodos sin acceso no controló diferencias en la disminución en la ingesta entre los periodos sin acceso. Es decir, alargar el periodo sin acceso a la comida parece tener efectos aditivos en el control del estímulo sobre la ingesta sólo para presentaciones del estímulo contiguas a la comida.

Finalmente, cabe mencionar que los resultados del presente estudio son pertinentes a la discusión relativa a cuáles son los factores que controlan el inicio de un periodo de alimentación y de la cantidad de comida ingerida. En diferentes trabajos se ha descrito que la ingesta de comida es controlada por la privación de comida y por la presentación de estímulos relacionados con comida (e.g., W eingarten, 1985; Zamble, 1973) no obstante, no se había explorado la manera en que estos factores interactúan para determinar la ingesta. Ahora se sabe que intervalos estímulo-comida 
cortos en combinación con periodos sin acceso a la comida largos son más conducentes a controlar aumentos en la ingesta de comida en comparación con periodos sin acceso cortos combinados con intervalos estímulo-comida largos.

\section{Referencias}

Ávila, R. \& Bruner, C. A. (1989). Efectos del intervalo respuesta-reforzador y del ciclo de reforzamiento en un programa de demora variable. Revista Mexicana de Análisis de la Conducta, 15(1), 23-39.

Baker, R. A. (1955). The effects of repeated experience on feeding behavior. Journal of Comparative and Physiological Psychology, 48, 37-42.

Bruner, C. A. (2010). Conducta de comer: variables comunes a través del condicionamiento y la motivación. Revista Mexicana de Análisis de la Conducta, 36(2), 1-11.

Díaz, F. (2008). Variables que controlan la ingesta de alimento en ratas: un enfoque paramétrico entre la teoría de la motivación y el análisis experimental de la Conducta (Tesis doctoral inédita). Universidad Nacional Autónoma de México, México.

Farmer, J. \& Schoenfeld, W. N. (1966a). Varying temporal placement of an added stimulus in a fixed-interval Schedule. Journal of the Experimental Analysis of Behavior, 9, 369-375.

Hernández, V., \& Bruner, C. (2012). El consumo de comida en ratas controlado por un estímulo neutral. Acta Comportamentalia, 20, 255-267.

Honig, W., Boneau, C., Burstein, K., \& Pennypacker, H. (1963). Positive and negative generalization gradients obtained after equivalent training conditions. Journal of Comparative and Physiological Psychology, 56, 111-116.

Lovibond, P. F. (1980). Effects of long- and variable-duration signals for food on activity, instrumental responding and eating. Learning and Motivation, 11, 164-184.

Nevin, J. (1973). Stimulus control. En J. Nevin \& G. S. Reynolds (Eds.). The Study of Behavior (pp. 114-152). Glenview, Illinois: Scott, Foresman and Company.

Pavlov, I. P. (1927). Conditioned reflexes. Oxford.: Oxford University Press.

Siegal, P. S. (1961). Food intake in the rat in relation to the dark-light cycle. Journal of Comparative and Physiological Psychology, 54, 294-301.

Schoenfeld, N. (1966). Conditioning the whole organism. Integrative Physiological and Behavioral Science, 31, 258-260.

Teff, K. (2000). Nutritional implications of the cephalic-phase reflexes: Endocrine responses. Appetite, 34, 206-2013.

Weingarten, H. P. (1985). Stimulus control of eating: Implications for a Two-factor theory of hunger. Appetite, 6, 387-401.

Zamble, E. (1973). Augmentation of eating following a signal for feeding in rats. Learning and Motivation, 4, 138-147. 